\title{
Características y utilidad de las Revisiones Sistemáticas o Meta-análisis
}

\author{
Elías Lessin García Alba
}

$\mathrm{E}^{\mathrm{n}}$ el ámbito de la investigación biomédica, cada día se van elaborando, desarrollando y publicando un número creciente de Artículos Originales con un sin número de tipos de variables, enfoques y resultados muy heterogéneos en algunos $\operatorname{casos}^{1,2}$.

Por esa razón es imprescindible y de mucha utilidad la existencia de revisiones sistemáticas (RS) o meta análisis que sinteticen y vuelvan homogénea la información relevante acerca de un tema en específico, optimizando la lectura de un numero enorme de Artículos sobre un tema puntual que en algunos casos tienen resultados muy heterogéneos, convirtiéndolo en un solo Artículo que ejemplifique lo mejor de cada uno ${ }^{1,3}$.

Entre las ventajas que nos proporcionan las revisiones sistemáticas podemos mencionar que nos presenta una síntesis de información respecto de una pregunta clínica específica, nos permite resumir en un valor numérico toda la evidencia relacionada a un tema puntual. Es considerada el primer nivel de evidencia dentro la pirámide de los niveles de evidencia (Ver tabla 1$)^{4,5}$, a raíz de estos estudios se puede plantear nuevas hipótesis para futuros estudios y también permite cuantificar la magnitud del sesgo de publicación ${ }^{1,2}$.

Entre las limitaciones se encuentran el hecho de que este tipo de estudios son retrospectivos por lo cual se pueden presentar sesgos durante el proceso de búsqueda, selección, análisis y síntesis de la información, para lo cual los autores deben tomar todas las previsiones para que esto no ocurra, también hay que tomar en cuenta que la realización de las revisiones sistemáticas o meta análisis está limitada por la cantidad y calidad de los estudios previamente realizados ${ }^{1,4}$.

Para poder reconocer una buena revisión sistemática se sugiere que respon- da por lo menos a estas 6 preguntas ${ }^{2}$, 4, 5 :

- ¿Aborda la revisión una pregunta clínica lógica y focalizada?

- ¿Fueron claros y apropiados los criterios para la inclusión y exclusión de estudios?

- ¿Es poco probable que se pasaran por alto estudios relevantes?

- ¿Se evaluó la validez de los estudios incluidos?

- ¿Fueron reproducibles las evaluaciones de los estudios?

- ¿Fueron consistentes los resultados de estudio a estudio?

La clave de todo este proceso de elaboración de una revisión sistemática o meta análisis radica en la correcta selección de artículos incluidos en el trabajo, ya que los autores se podrían limitar solo a artículos publicados en las más grandes bases de datos, dejando de lado los trabajos existentes en jornadas de congresos, jornadas científicas o tesis, para los cuales los autores deben ser capaces de reunir o incluir en cierta manera ese gran número de trabajos que no fueron publicados ${ }^{2,5,6}$; en este entendido es imperiosa la necesidad de elaborar los instrumentos de búsqueda y selección de artículos correctamente, delimitar y especificar las variables codificadas de los estudios (para una mejor sistematización de la información y depuración rápida de artículos sin mucha relevancia para el estudio) y contar con los métodos estadísticos correctos $^{2,5,6}$; además se debe tratar de buscar trabajos de investigación que no fueron publicados en revistas o bases de datos electrónicas (para evitar el sesgo en la selección de artículos), llamando al comité organizador de congresos, hospitales o instituciones que podrían albergar información importante para el estudio ${ }^{2,5}$.

Cuando se consigue que un meta análisis tenga las características antes descritas y se tenga la certeza de haber eli- minado la gran mayoría de los posibles sesgos, entonces se puede afirmar que se elaboró una herramienta de mucha utilidad para la comunidad científica, ya que las conclusiones de los meta análisis al englobar un numero bastante grande de estudios, sus conclusiones son más fiables y generalizables que las de los estudios individuales ${ }^{1,2}$.

Elías Lessin García Alba ${ }^{1}$

${ }^{1}$ Director del comité Científico de la Revista Científica Ciencia Médica, Estudiante de Medicina de la Facultad de Medicina-Universidad Mayor de San

Simón, Cochabamba-Bolivia

Correspondencia a:

lessin229@gmail.com

\section{Referencias:}

1. Letelier LM, Moore P. La Medicina Basada en Evidencia, visión después de una década. Rev. Med Chile 2003; 131: 939-46.

2. Letelier LM, Manríquez JJ, Rada G. Revisiones sistemáticas y meta análisis: ¿son la mejor evidencia? Rev. Med Chile 2005; 133: 246-249.

3. Urrútia G, Bonfill X. Declaración PRISMA: una propuesta para mejorar la publicación de revisiones sistemáticas y meta análisis. Med Clin (Barc). 2010; 135(11): 507-511.

4. Manterola C, Zavando D. Como interpretar los "Niveles de Evidencia" en los diferentes escenarios clínicos. Rev. Chilena de Cirugía. 2009;61(6):582-95.

5. Sanchez-Meca J, Botella J. Revisiones sistemáticas y Meta-análisis: Herramientas para la práctica profesional. Papeles del Psicólogo. 2010;31(1):7-17

6. Sanchez-Meca J, Marin-Martinez F, LopezLopez JA. Meta-análisis e Intervención Psicosocial Basada en la Evidencia. Psychosocial Intervention 2011;20(1):95-107 


\begin{tabular}{|c|c|c|c|c|c|}
\hline Recomendación & Nivel & $\begin{array}{l}\text { Terapia prevención, } \\
\text { etiología y daño }\end{array}$ & Pronóstico & Diagnóstico & Estudios económicos \\
\hline \multirow{3}{*}{ A } & $1 a$ & $\begin{array}{l}\text { RS con homogeneidad y } \\
\text { Meta-análisis de EC }\end{array}$ & $\begin{array}{l}\text { RS con homogeneidad y Meta-análisis de } \\
\text { estudios de cohortes concurrente }\end{array}$ & RS de estudios de diagnostico nivel 1 & RS de estudios económicos de nivel 1 \\
\hline & $1 b$ & $\begin{array}{l}\text { EC individuales con } \\
\text { intervalo de confianza } \\
\text { estrecho }\end{array}$ & $\begin{array}{l}\text { Estudio individual de cohorte } \\
\text { concurrente con seguimiento superior al } \\
80 \% \text { de la cohorte }\end{array}$ & $\begin{array}{l}\text { Comparación independiente y } \\
\text { enmascarada de un espectro de } \\
\text { pacientes consecutivos sometidos a } \\
\text { la prueba diagnóstica y al estándar } \\
\text { de referencias }\end{array}$ & $\begin{array}{l}\text { Análisis que compara los desenlaces } \\
\text { posibles, contra una medida de costos. } \\
\text { Incluye un análisis de sensibilidad }\end{array}$ \\
\hline & $2 a$ & $\begin{array}{l}\text { RS con homogeneidad } \\
\text { de estudios de cohortes }\end{array}$ & RS de cohortes históricas & $\begin{array}{l}\text { RS de estudios diagnósticos de nivel } \\
\text { mayor a } 1\end{array}$ & $\begin{array}{l}\text { RS de estudios económicos de nivel } \\
\text { mayor a } 1\end{array}$ \\
\hline \multirow[t]{3}{*}{ B } & $2 b$ & $\begin{array}{l}\text { Estudio de cohortes } \\
\text { individual EC de baja } \\
\text { calidad }\end{array}$ & Estudio individual de cohorte históricas & $\begin{array}{l}\text { Comparación independiente } \\
\text { enmascarada de pacientes no } \\
\text { consecutivos, sometidos a la prueba } \\
\text { diagnóstica y al estándar de } \\
\text { referencias }\end{array}$ & $\begin{array}{l}\text { Comparación de un número limitado de } \\
\text { desenlaces contra una medida de costo. } \\
\text { Incluye análisis de sensibilidad }\end{array}$ \\
\hline & $3 a$ & $\begin{array}{l}\text { RS con homogeneidad } \\
\text { de estudios de casos y } \\
\text { controles }\end{array}$ & & & \\
\hline & $3 b$ & $\begin{array}{l}\text { Estudio de casos y } \\
\text { controles individuales }\end{array}$ & & $\begin{array}{l}\text { Estudios no consecutivos o carentes } \\
\text { de un estándar de referencia }\end{array}$ & $\begin{array}{l}\text { Análisis sin una medida exacta de costo, } \\
\text { pero incluye análisis de sensibilidad }\end{array}$ \\
\hline C & 4 & $\begin{array}{l}\text { Series de casos. Estudios } \\
\text { de cohortes y casos y } \\
\text { controles de mala } \\
\text { calidad }\end{array}$ & $\begin{array}{l}\text { Series de casos. Estudios de cohortes de } \\
\text { mala calidad }\end{array}$ & $\begin{array}{l}\text { Estudios de casos y controles sin la } \\
\text { aplicación de un estándar de } \\
\text { referencia }\end{array}$ & Estudio sin análisis de sensibilidad \\
\hline D & 5 & $\begin{array}{l}\text { Opinión de expertos sin } \\
\text { evaluación critica } \\
\text { explicita, o basada en } \\
\text { fisiología, o en } \\
\text { investigación teórica. }\end{array}$ & $\begin{array}{l}\text { Opinión de expertos sin evaluación critica } \\
\text { explicita, o basada en fisiología, o en } \\
\text { investigación teorica }\end{array}$ & $\begin{array}{l}\text { Opinión de expertos sin evaluación } \\
\text { critica explicita, o basada en } \\
\text { fisiología, o en investigaciones } \\
\text { teoricas }\end{array}$ & $\begin{array}{l}\text { Opinión de expertos sin evaluación } \\
\text { critica explicita, o basada en teoría } \\
\text { económica }\end{array}$ \\
\hline
\end{tabular}

Tabla 1: Clasificación de los niveles de evidencia según Sackett ${ }^{4}$

*Por Homogeneidad se entiende RS que está libre de variaciones (heterogenei-

dad) en las direcciones o grados de resultados entre los estudios individuales.

Información para el autor: Búsqueda de palabras MeSH

1. Ingresa a: http://www.nlm.nih.gov/mash/Mbrowser.html

2. Introducir la palabra clave que se quiera comprobar

3. Seleccionar "Descriptor"

4. Se indicará si existe o no la palabra clave seleccionada 